\title{
Effectiveness of Mindfulness-Based Happiness Pregnant Birth Parenting (HPBP) Programs on Mental Health During Pregnancy and Early Motherhood in China: A Multicenter Randomized Control Trial
}

\section{Wang Shulei}

National Center for Women and Children's Health, China CDC

\section{Sun Mengyun}

National Center for Women and Children's Health, China CDC

Huang Xing

National Center for Women and Children's Health, China CDC

\section{Zhang Daming}

Shanxi Maternal and Child Health Hospital

\section{Yang Li}

National Center for Women and Children's Health, China CDC

Xu Tao

National Center for Women and Children's Health, China CDC

Pan XiaoPing

National Center for Women and Children's Health, China CDC

Zheng Ruimin ( $\square$ zhengruimin@chinawch.org.cn )

National Center for Women and Children's Health, China CDC

\section{Research Article}

Keywords: mindfulness, depression, anxiety, fear, life satisfaction, randomized controlled trial

Posted Date: January 27th, 2022

DOI: https://doi.org/10.21203/rs.3.rs-1126757/v1

License: (a) (i) This work is licensed under a Creative Commons Attribution 4.0 International License. Read Full License 


\section{Abstract \\ Background}

This study aimed to investigate the efficacy of Happiness Pregnant Birth Parenting (HPBP) in preventing perinatal depression and reducing perceived anxiety and fear in Chinese pregnant women.

\section{Methods}

Women experiencing first-time pregnancy $(n=104)$ were randomly allocated to the HPBP group or a parallel control treatment group; We collected data at baseline (T0), post-intervention ( $T 1$ ), three days after delivery (T2), and 42 days after delivery (T3). Participants completed questionnaires regarding depressive symptoms, perceived anxiety, fear, satisfaction with life, and five facets of mindfulness. We analyzed differences between the two groups and changes within the same group at the four time points using repeated-measures ANOVA.

\section{Results}

Compared to the active control treatment group, the HPBP group reported lower levels of perceived depressive symptoms at T2 $(p<.05, d=1.83)$ and T3 $(p=.01, d=2.21)$; significantly reduced anxiety at $\mathrm{T} 1(p=.001, d=5.31)$ and T2 $(p=.003, d=6.12)$; significantly reduced fear at T1 $(p<.01, d=14.95)$ and T2 $(p=.04, d=10.95)$; and significantly increased life satisfaction at T1 $(p<.01, d=3.86)$ and T3 ( $p=$ $.015, d=3.1)$ and self-reported mindfulness at T1 $(p=.01, d=6.81)$ and T2 $(p<.01, d=8.7)$.

\section{Conclusions}

The findings indicate that the HPBP intervention based on mindfulness effectively decreases the risk of perinatal depression, anxiety, and fear. Thus, this research enhances our knowledge of effective intervention strategies to promote mental well-being and prevent perinatal depression or other negative mental states among pregnant women.

\section{Trial registration}

:Chinese Clinical Trial Registry (ChiCTR):ChiCTR2000033149; The date of first registration is 24/ 05/ 2020.

\section{Background}


The transition to motherhood is a stressful phase that requires women to make immediate and significant changes in their daily lives, including internal thinking and external behavior [1]. This major lifestyle shift can be fraught with difficulties and extra stress, leaving new mothers prone to depression, anxiety, and other negative emotions. Pregnant women have higher levels of depression and anxiety than the general population, and the most common complication of childbearing is postnatal depression [2]. A meta-analysis reviewed 56 countries found that $17.7 \%$ of pregnant women worldwide suffer postpartum depression (PPD) [3]. According to Sun et al., the National Maternal and Child Health Institution of China confirmed that the detection rate of anxiety among pregnant women during early pregnancy in China was $19.9 \%$ [4]. In addition, fear of childbirth is a common psychological problem among mothers-to-be. Maeve et al. conducted a systematic review and meta-analysis study that showed that the global prevalence of childbirth fear among pregnant women was $14 \%$ [5].

Depression, anxiety, and fear influence each other and predict poor labor and delivery related outcomes. Increasing evidence has shown that perinatal depression and anxiety can negatively affect new mothers' personal and social lives, physical health, and interpersonal communication and reduce their attachment to their newborns. Perinatal depression and anxiety also predict long- and short-term negative consequences on the physical and mental development of newborns[6]. The Apgar score (Activity, Pulse, Grimace, Activity, Respiration) is a standard assessment for a newborn's physical condition immediately after birth. The lower the score, the worse the child's condition. However, low Apgar scores, fetal growth retardation, and preterm birth were associated with depression and anxiety in the short term $[7,8]$. Over the long term, lasting perinatal anxiety and depression can increase the risk of children's abnormal neurobehavioral and cognitive development [9]. Fear of childbirth poses a significant risk to the health of both mother and child, especially from pregnancy to childbirth and in the early postpartum period. It leads to low childbirth self-efficacy, increased unwanted obstetric interventions, increased use of pain medication during childbirth [10-12], and increased risk of PPD [13]. Using data from more than 25,000 pregnant women in the Danish National Birth Cohort, Laursen et al. found that pregnant women's fear of childbirth increased the risk of emergency c-sections during the third trimester of pregnancy (approximately 31 weeks) [14].

While transitioning to motherhood, women continue to shoulder a heavy workload as well as meet social and family responsibilities while caring for their newborns. This transition can be challenging. Therefore, women's mental health deserves particular attention. To prevent or attenuate mental distress among pregnant women should be a crucial public health goal [15]. Helpful psychological interventions during pregnancy are urgently needed, and women, especially first-time mothers, require organized antenatal education and preparation for birth.

In the previous two decades, mindfulness-based programs have gradually become more popular to help people improve their well-being, and a mindfulness-based childbirth and parenting (MBCP) program [16, 17] was developed for pregnant women in the United States, adopted by Bardacke from mindfulnessbased stress reduction (MBSR) therapy [18]. MBSR therapy aims to teach pregnant women and their partners' mindfulness skills to manage anxiety and depression during pregnancy, cope with fear and pain 
during childbirth, and foster sensitive parenting [19]. Studies in many countries have shown that MBCP programs can effectively alleviate pregnant women's anxiety, depression, fear of childbirth, and other negative emotions and facilitate childbirth self-efficacy and marital satisfaction [20-25].

Chinese culture is different from Western culture. So far, there are no studies on the effects of mindfulness interventions on pregnant women in China. To both increase the MBCP program's compatibility with Chinese culture and meet the needs of pregnant women in China, Dr. Zheng from the National Center for Women and Children's Health arranged for renowned experts from China and other countries to adjust the MBCP curriculum locally in China and subsequently developed the Happiness Pregnant Birth Parenting (HPBP) curriculum [26]. The course format examined in the current study was 21 days online and four days on-site. We explore the psychological effects of localized MBCP courses on pregnant women in China for the first time. This study aimed to explore the efficacy of the HPBP program on Chinese pregnant women's depression, anxiety, fear, life satisfaction, and mindfulness. Preventive HPBP interventions were designed to promote a positive birth experience and alleviate psychological problems for women.

\section{Methods}

\section{Study design and sample size}

This study was a multicenter randomized trial and parallel design. G*Power 3.1 network-based software was adopted to calculate the sample size; reference to the effect size was adopted from Goyal et al. $(\mathrm{a}=$ 0.05 , power $=0.90$ ) [27]. At least 74 participants totally were needed for the study. The study was conducted from September 2018 to April 2020 at a regional hospital of Shanghai, Shijiazhuang, and Sichuan provinces.

We created random grouping sequences using SAS software and assigned participants to a control group or an intervention group in a 1:1 ratio based on their time of enrollment.

\section{Participants}

Inclusion criteria: pregnant women with a single pregnancy who were currently at 20-32 weeks of gestation, able to communicate fully in Chinese, and had visited an obstetrician at the appointed hospital during the recruiting. Furthermore, the participants had to have a high school education or above and be without serious pregnancy complications or diseases. Pregnant women diagnosed with psychosis who were undergoing any form of psychological therapy or were taking psychotropic medications were excluded. We also excluded pregnant women who have had a repeated abortion, preterm birth, or a history of epilepsy., We initially selected 104 women to participate in the study based on the inclusion criteria, as shown in Figure 1.

\section{Interventions}


The experimental group participated in the HPBP course introduced by Dr. Zheng. The course provided participants with self-awareness training based on MBSR using transformational experiences related to pregnancy, childbirth, and postnatal adjustment. The intervention group followed the course format of 4 days of on-site teaching combined with 21 days of online teaching. This group received the HPBP intervention in group settings during weekends for four consecutive weeks. Intervention courses were taught by two researchers who have completed professional training. In addition, during the intervention, we arranged for two other researchers to observe and guide the participants in the course, communicate, and provide feedback after the course. We encouraged the participants to accomplish the mindfulness exercises during classes and practice at home using the Whale Punch app for 21 days with the recorded audio. Mindfulness-based psychosomatic interventions for pregnant women mainly included raisin exercise, breath awareness, body scan, mindful yoga and meditation, labor pain cognitive education, and pain management with ice water exercises.

We conducted conventional childbirth education at home using the Whale Punch app for the participants in the control group for 21 days, with the recorded audio. The course covered physical and psychological information related to pregnancy as well as self-care skills during pregnancy and postpartum.

\section{Data collection and measures}

We collected the questionnaires before (T0) and after the intervention (T1), three days after delivery (T2), and 42 days after delivery (T3) at the hospital clinic. All participants completed the Edinburgh Postnatal Depression Scale (EPDS), the Self-rating Anxiety Scale (SAS), the Delivery Expectancy/Experience Questionnaire (W-DEQ-A/B), the Satisfaction with Life Scale (SWLS), and the Five Facet Mindfulness Questionnaire (FFMQ) at each self-report measure administration.

\section{EPDS}

This questionnaire includes ten items. Each item was scored from 0 to 3 , and we added up the scores for each item to get the total[28]. A prenatal EPDS score greater than 13 was considered an indicator of depression [29]; Tsao et al. previously used a cutoff value of 13 in a study involving Taiwanese women [30]. Cronbach's alpha was 0.85 in our study.

\section{FFMQ}

The FFMQ is based on factor analysis and includes 39 questions and five factors; observing, describing, acting with awareness, being non-judgmental of inner experience, and being non-reactive to inner experience. Responses were scored using a five-point Likert-type scale, with one representing "never or very rarely true" and five representing "very often or always true" [31]. Cronbach's alpha was 0.85 in our study.

\section{SWLS}


The SWLS [32] is a five-item self-report scale that primarily comprises questions concerned with personal life satisfaction. Responses are scored on a seven-point Likert-type scale ( 1 = "strongly disagree" and 7 = "strongly agree"). The internal consistency of the scale is 0.78 , and Cronbach's alpha is 0.79 in the present study.

\section{W- DEQ-(A/B)}

The W-DEQ-A/B measures women's expectations about childbirth before (version A) and after (version B) delivery to assess fear of childbirth during pregnancy and after childbirth. There are 33 items scored using a six-point Likert-type scale, with 0 representing "extremely" and 5 representing "not at all." The total score is between 0 and 165, and the higher the score, the higher the fear level [33]. Cronbach's alpha for questionnaires $A$ and $B$ were 0.93 and 0.94 , the half-fold reliability was 1.0 and 0.95 , and the calibration correlation validity was more significant than or equal to 0.82 , respectively [34].

\section{SAS}

The SAS [35] is a self-report scale with 20 items covering a variety of psychological and somatic anxiety symptoms. Participants responded on a four-point scale ( $1=$ "none, or little time" to $4=$ "most or all the time"). Participants answered questions based on their experiences from the previous week. The original SAS score ranges from 20 to 80 . SAS has shown satisfactory psychometric performance [36], and Cronbach's alpha in the present study was 0.83 .

\section{Ethical considerations}

The study protocol was approved by the Ethics Review Committee of the National Center for Women and Children (Chinese Center for Disease Control and Prevention, Beijing, China; approval no. FY2018-30). Before recruitment, we explained the purpose, significance, benefits, and potential risks of our study in detail to every pregnant woman who was interested in participating in the study. Additionally, we informed the participants regarding what they needed to do and what was forbidden in our study and follow-up to obtain their understanding and support. The participants provided informed consent by signing informed consent forms. In addition, to keep data confidential, all data collected were anonymous and prohibited for use outside of the present research. We informed the participants that they had the right to exit from participation at any time in the study period with no consequences.

\section{Data analysis}

SPSS 24.0 was used for data analysis. We summarized demographic characteristics as the mean (standard deviation [SD]) for measurement data and as frequency counts (percentages) for categorical variables. We used the chi-square test and Fisher's exact test to evaluate the differences among the three groups of demographic variables (education level, marital status, and family income). Rank scores and measurement data were analyzed using analysis of variance (ANOVA). Longitudinal data were analyzed using repeated-measures ANOVA to measure the result variables $\mathrm{T} 0, \mathrm{~T} 1, \mathrm{~T} 2$, and $\mathrm{T} 3$. The person in charge of the data analysis did not know the grouping until the data was analyzed. 


\section{Results}

In total, we interviewed 104 participants, of which six were eliminated because they did not meet the criteria for inclusion. Another 15 participants were excluded from further analysis because of their unwillingness to participate. There were no statistically significant differences between the ultimate group of participants $(n=83)$, including the intervention $(n=40)$ and comparison $(n=43)$ groups and those subjects that dropped out $(n=15)$ in terms of age, infant's gestational age, body weight, education level, census register, marital status, household income, parity, pregnancy method, and pregnancy complications (Table 1, Figure 1).

Table 1 Characteristics of participants 


\begin{tabular}{|c|c|c|c|c|c|c|}
\hline Characteristics & All(98) & $\begin{array}{l}\text { Intervention } \\
\text { group(40) }\end{array}$ & $\begin{array}{l}\text { Comparison } \\
\text { group(43) }\end{array}$ & $\begin{array}{l}\text { Lost to } \\
\text { follow } \\
\text { up(15) }\end{array}$ & Statistics & $\mathbf{P}$ \\
\hline Age $(M \pm S D)$ & $31.75 \pm 3.2$ & $31.83 \pm 3.5$ & $31.51 \pm 3$ & $32.1 \pm 2.5$ & 0.29 & $0.75^{b}$ \\
\hline $\begin{array}{l}\text { Gestational age of } \\
\text { infant }(M \pm S D)\end{array}$ & $26.38 \pm 4.0$ & $26.23 \pm 3.8$ & $27 \pm 4.1$ & $25.2 \pm 4.0$ & 1.16 & $0.32^{b}$ \\
\hline Bodyweight(M $\pm S D)$ & $64.1 \pm 8.85$ & $63.19 \pm 8.4$ & $6.15 \pm 9.15$ & $61.8 \pm 8.8$ & 1.87 & $0.16^{\mathrm{b}}$ \\
\hline Level of education & & & & & 1 & $0.61^{a}$ \\
\hline $\begin{array}{l}\text { Junior college or } \\
\text { below }\end{array}$ & 19 & 7 & 10 & 2 & & \\
\hline University or above & 80 & 33 & 33 & 14 & & \\
\hline Census register & & & & & 0.42 & $0.81^{a}$ \\
\hline Urban & 61 & 26 & 25 & 10 & & \\
\hline Rural & 38 & 14 & 18 & 6 & & \\
\hline Marital status & & & & & 1.23 & $0.56^{c}$ \\
\hline Married & 96 & 39 & 42 & 15 & & \\
\hline Not married & 3 & 1 & 1 & 1 & & \\
\hline Income & & & & & 5.34 & $0.25^{a}$ \\
\hline Less than $¥ 100,000$ & 27 & 13 & 11 & 3 & & \\
\hline$¥ 100,000-¥ 200,000$ & 39 & 15 & 20 & 4 & & \\
\hline More than $¥ 200,000$ & 33 & 12 & 12 & 9 & & \\
\hline Parity & & & & & 1.95 & $0.38^{a}$ \\
\hline No prior births & 75 & 32 & 33 & 10 & & \\
\hline 1 or more prior births & 24 & 8 & 10 & 6 & & \\
\hline Pregnancy way & & & & & 0.53 & $1^{\mathrm{c}}$ \\
\hline pregnancy by nature & 94 & 38 & 41 & 14 & & \\
\hline $\begin{array}{l}\text { Pregnancy by } \\
\text { medicine }\end{array}$ & 5 & 2 & 2 & 1 & & \\
\hline
\end{tabular}

a Chi-square test, X2; b Analysis of variance, ANOVA; c Fisher's exact test. Significant at the 0.05 level. 


\begin{tabular}{|lllllll|}
\hline Characteristics & All(98) & $\begin{array}{l}\text { Intervention } \\
\text { group(40) }\end{array}$ & $\begin{array}{l}\text { Comparison } \\
\text { group(43) }\end{array}$ & $\begin{array}{l}\text { Lost to } \\
\text { follow } \\
\text { up(15) }\end{array}$ & Statistics & P \\
\hline $\begin{array}{l}\text { pregnancy } \\
\text { complications }\end{array}$ & 72 & 33 & 30 & 13 & 2.1 & $0.35^{\text {a }}$ \\
\hline No & 27 & 7 & 13 & 3 & \\
\hline Yes & & & & & \\
\hline a Chi-square test, X2; $b$ Analysis of variance, ANOVA; c Fisher's exact test. Significant at the 0.05 level. \\
\hline
\end{tabular}

Table 2 Assessing the effects of depression, anxiety, fear, satisfaction with life, and mindfulness using repeated measures analysis of variance 


\begin{tabular}{|c|c|c|c|c|c|}
\hline Variable & group & TO & T1 & T2 & T3 \\
\hline \multirow[t]{2}{*}{ EPDS } & $\begin{array}{l}\text { intervention } \\
\text { group }\end{array}$ & $14.1 \pm 3.62$ & $13.42 \pm 3.41$ & $12.12 \pm 4.38^{* a b}$ & $12.2 \pm 4.06^{* a}$ \\
\hline & control group & $13.74 \pm 3.44$ & $13.79 \pm 3.15$ & $13.95 \pm 4.01$ & $14.4 \pm 3.86$ \\
\hline \multirow[t]{2}{*}{ SAS } & $\begin{array}{l}\text { intervention } \\
\text { group }\end{array}$ & $41.24 \pm 8.13$ & $40.11 \pm 6.35^{*}$ & $37.51 \pm 5.83^{* a}$ & $39.46 \pm 9.91$ \\
\hline & control group & $41.44 \pm 6.6$ & $45.41 \pm 6.99^{a}$ & $43.63 \pm 10.64$ & $43.46 \pm 11.09$ \\
\hline \multirow[t]{2}{*}{$\begin{array}{l}\text { W-DEQ- } \\
\text { A/B }\end{array}$} & $\begin{array}{l}\text { intervention } \\
\text { group }\end{array}$ & $72.19 \pm 22.15$ & $60.79 \pm 18.53^{* a}$ & $62 \pm 24.94^{* a}$ & N/A \\
\hline & control group & $70.78 \pm 18.04$ & $75.73 \pm 17.83$ & $72.95 \pm 22.49$ & $\mathrm{~N} / \mathrm{A}$ \\
\hline \multirow[t]{2}{*}{ SWLS } & $\begin{array}{l}\text { intervention } \\
\text { group }\end{array}$ & $25.98 \pm 3.98$ & $28.35 \pm 3.99^{* a}$ & $\mathrm{~N} / \mathrm{A}$ & $25.58 \pm 5.4^{* b}$ \\
\hline & control group & $24.78 \pm 5.48$ & $24.47 \pm 4.04$ & $\mathrm{~N} / \mathrm{A}$ & $22.47 \pm 5.74^{\mathrm{ab}}$ \\
\hline \multirow[t]{2}{*}{ FFMQ } & $\begin{array}{l}\text { intervention } \\
\text { group }\end{array}$ & $131 \pm 12.16$ & $133.22 \pm 11.42^{*}$ & N/A & $131.49 \pm 15.75^{*}$ \\
\hline & control group & $129.77 \pm 11.46$ & $126.41 \pm 12.16$ & N/A & $122.79 \pm 11.9^{a}$ \\
\hline \multicolumn{6}{|c|}{$\begin{array}{l}\mathrm{T} 0=\text { before the intervention; } \mathrm{T} 1=\text { after the intervention; } \mathrm{T} 2=3 \text { days after delivery; } \mathrm{T} 3=42 \text { days after } \\
\text { delivery. }\end{array}$} \\
\hline \multicolumn{6}{|c|}{$\begin{array}{l}\text { EPDS, Edinburgh Postnatal Depression Scale; FFMQ, Five Facet Mindfulness Questionnaire; SAS, Self- } \\
\text { rating Anxiety Scale; SWLS, Satisfaction with Life Scale; W-DEQ-A/B, Delivery Expectancy/Experience } \\
\text { Questionnaire. }\end{array}$} \\
\hline \multicolumn{6}{|c|}{ * There was a statistical difference between the intervention and control groups. } \\
\hline \multicolumn{6}{|c|}{$\begin{array}{l}\text { a Compared with } \mathrm{T} 0 \text {, there was a statistical difference; }{ }^{\mathrm{b}} \text { Compared with } \mathrm{T} 1 \text {, there was a statistical } \\
\text { difference; }{ }^{c} \text { Compared with } \mathrm{T} 2 \text {, there was a statistical difference. }\end{array}$} \\
\hline
\end{tabular}

The longitudinal data analysis results are presented in Table 2 and Figure2. Regarding depression, the means of the two groups were compared at the same time points. At T0, the average score of the intervention group was 0.36 points higher than the control group, with no statistical significance $(p=$ 0.65). After the intervention, the average score of the intervention group was lower than that of the control group, with a difference of 0.37 points $(p=0.61)$. The difference between the two groups gradually emerged over time: at T2 and T3, it was 1.83 points $(p<0.05)$ and 2.21 points $(p=0.01)$, respectively. A comparison of mean values at different time points within the group showed that there was no statistical significance between the four time points in the control group; however, in the intervention group, the mean scores at T2 and T3 were significantly lower than at T0. Additionally, the mean scores at T2 were significantly lower than at T1. 
For anxiety, the comparison of the means of the two groups at the same time indicated that the intervention group's average score was 0.2 points lower than that of the control group at T0, with no statistical significance $(p=0.91)$. After the intervention, the difference between the two groups widened to 5.31 points $(p=0.001)$ and 6.12 points $(p=0.003)$ at T2. However, the difference between the two groups narrowed to 4 points at T3 $(p=0.1)$. Mean comparisons of within-group scores at different time points indicated that in the control group, the mean scores at T1 were significantly higher than those at T0; however, in the intervention group, the mean scores at T2 were significantly lower than those at T0.

On the W-DEQ-A/B, the mean score for the experimental group was 1.41 points lower than that of the control group at T0 ( $p=0.91)$. The mean score for the experimental group was 14.95 points lower ( $p<$ 0.01) than that of the control group at T1; however, the gap between the two groups narrowed at $\mathrm{T} 2$, and the mean score for the experimental group was 10.95 points lower $(p=0.04)$ than that of the control group. The mean comparison of scores with groups at different time points indicated that the difference between the three time points in the control group was not statistically significant; however, in the experimental group, the mean scores at T1 and T2 were significantly lower than those at T0.

Regarding satisfaction with life, the average score of the intervention group at T0 was only 1.2 points lower than that of the control group $(p=2.66)$. After the intervention, the difference between the two groups widened to 3.86 points $(p<0.01)$. At T3, the difference between the two groups narrowed to 3.1 points $(p=0.015)$. Mean comparisons of within-group scores at different time points indicated those in the control group, the mean scores at T3 were significantly lower than those at T0 and T1. In contrast, in the intervention group, the mean scores at T1 were significantly higher than those at T0, and the mean scores at T3 were significantly higher than those at T1.

In the mindfulness questionnaire, the mean score of the intervention group at T0 was 1.23 points higher than the control group $(p=0.64)$. After the intervention, the difference between the two groups widened to 6.81 points $(p=0.01)$, and at T3, the difference widened to 8.7 points $(p<0.01)$. Within groups, mean comparisons indicated that in the control group, the mean scores at T3 were significantly lower than those at T0, and the difference among the three time points in the intervention group was not significant.

\section{Discussion}

A total of 83 pregnant women completed the study. The between-group difference in demographic characteristics and psychological measures was not statistically significant before the intervention. After the intervention, self-reported fear, anxiety, and depression were significantly reduced in the intervention group compared with the control group. During the intervention, participants in the intervention group learned mindfulness skills that allowed them to maintain lower levels of anxiety and depression and higher levels of life satisfaction and mindfulness than the participants in the control group, at least 42 days after delivery.

In the emerging field of prenatal MBCP research, data on long-term effects are rare, and few related studies are available. Gunilla et al. conducted a pilot randomized controlled trial (RCT) in which 193 
healthy pregnant women were randomly divided into two groups. The intervention group received training for eight weeks in the MBCP program, and the women's outcomes were followed up at baseline, postintervention, and at 3, 9, and 12 months postpartum. Researchers found that the intervention significantly decreased their EPDS scores and improved their FFMQ scores from baseline to post-intervention, but these effects did not persist at long-term follow-up assessments at 3, 9, and 12 months postpartum. This phenomenon was more pronounced among women who did not continue practicing after receiving the mindfulness intervention [37].In contrast to Gunilla et al.'s study, Miklowitz et al. found that after the mindfulness intervention, women maintained lower levels of depression up to 6 months postpartum [38].Furthermore, Luberto et al. conducted a single-arm study and found that the lower level of anxiety from pre- to post-intervention lasted up to three months after delivery, and reductions in depressive symptoms from pre- to post-intervention were further reduced at three months after delivery [39]. A randomized controlled study by Vieten and Astin showed that MBCP programs reduced state anxiety and negative emotions at 6 and 9 months of pregnancy in the intervention group [40]. This study found that lower levels of depressive and anxiety symptoms and high levels of mindfulness were sustained for at least 42 days postpartum. However, the effect of the intervention on anxiety decreased from 3 to 42 days postpartum. This may be because participants were not encouraged to continue their mindfulness training after the intervention cycle and the real life transitions that occurred postpartum.

With regard to fear, Duncan et al. conducted a pilot RCT with a small sample $(n=30)$ [41]. The intervention they applied was the short form of the nine-week MBCP program, and They measured fear of childbirth by W-DEQ. Similar to our study, they found that MBCP-based mindfulness training specifically targeted labor fear reduced labor fear post-intervention. However, in contrast to our study, the effect lasted until postpartum. This may be because Duncan's interventions targeted fear of childbirth and were given for a more extended period. Furthermore, Duncan and Bardacke [42], Veringa et al. [43], and Byrne et al. [44] found that Mindfulness interventions were effective in reducing fear of childbirth, which our findings support. Finally, Veringa-Skiba et al. found that to reduce just one woman's fear of childbirth to normal levels, MBCP training needs to be provided to at least five pregnant women [45].

Regarding mindfulness, Byrne et al. found that postpartum mindfulness increased between 3 and 12 weeks after delivery in a pre-and post-partum design with 18 participants [46]. In this study, the differences between the two groups were significantly different at 3 and 42 days after delivery. Moreover, the differences were more significant 42 days after delivery than at three days, indicating that the mindfulness intervention's effects continued for at least 42 days postpartum. Finally, Kantrowitz-Gordon et al. [47] conducted a qualitative study with a convenience sample and found that mindfulness exercises helped new mothers switch roles and find joy in early parenting. The participants also discovered the benefits of living in the present, which can improve family relationships, help them empathize with their partners, and be more likely to experience happiness, which, in turn, can increase life satisfaction and happiness; this is consistent with our research.

\section{Limitations}


In the present study, our result variables were measured by self-report questionnaires.

It is difficult to avoid the risk of reporting bias. Further, the women were not blinded to treatment conditions. Another limitation is that the participants were predominantly highly educated urban residents; therefore, evaluating the program in a more diverse population is necessary.

\section{Conclusions}

The current study results show that the HPBP program has a certain effect on improving Chinese pregnant women's physical and mental health. However, moderate effect sizes were observed from 3 days to 42 days after delivery in anxiety, depression, life satisfaction, and fear. Nevertheless, as it is necessary to consider small to medium effect sizes in pre- to post-analysis research on mindfulness [48], these results should be considered as this study examined whether these identified differences persisted up to 42 days postpartum in mental health. Furthermore, both groups reported a slight increase in depression and anxiety and decreased life satisfaction and mindfulness levels at 42 days after delivery. Postpartum intervention should be continued to ensure that the beneficial effects of mindfulness intervention are sustained [49].

The present study further demonstrates that mindfulness intervention effectively improves mental health in different perinatal populations, which is consistent with previous studies [50-52]. Additionally, this study is the first exploration of the psychological effects of localized MBCP courses on pregnant women in China. Furthermore, few previous studies have assessed the effects of prenatal interventions until 42 days postpartum (T3). As such, this study adds to our knowledge of effective intervention strategies to improve mental health and prevent perinatal depression in pregnant women.

A long-term longitudinal study is necessary to validate and further explore the effects of mindfulness on mothers and their children. It would be interesting to assess whether the advantageous effects of HPBP programs on mental health will persist over time, whether they are applied to more diverse populations, and whether there are any beneficial effects on childbirth self-efficacy, coping with the stress of pregnancy, coping with the pain of delivery, the long-term health outcomes of children, breastfeeding, and behavior efficacy measures such as the sense of attachment between mother and child. In addition, we should further explore the curriculum model adapted to China and identify more suitable groups for intervention to improve the efficiency of the interventions. The mechanism behind the effect of mindfulness intervention also needs to be studied.

\section{List Of Abbreviations}

ANOVA

analysis of variance

EPDS

Edinburgh Postnatal Depression Scale 
FFMQ

Five Facet Mindfulness Questionnaire

HPBP

Happiness Pregnant Birth Parenting

MBCP

mindfulness-based childbirth and parenting program

MBSR

mindfulness-based stress reduction

PPD

postpartum depression

RCT

randomized controlled trial

SAS

Self-rating Anxiety Scale

SWLS

Satisfaction with Life Scale

W-DEQ-A/B

Delivery Expectancy/Experience Questionnaire

\section{Declarations}

\section{Ethics approval and consent to participate}

The study protocol was approved by the Ethics Review Committee of the National Center for Women and Children's Health (Chinese Center for Disease Control and Prevention, Beijing, China; approval no. FY202010) and conducted in accordance with the ethical principles regarding human experimentation of the Declaration of Helsinki. All participants submitted signed informed consent prior to inclusion in the study; We promise that they can terminate their participation for any reason at any time.

\section{Consent for publication}

Not applicable.

\section{Availability of data and materials}

The data sets used in this study are available from the corresponding authors upon reasonable request.

\section{Competing interests}

The authors declare that there are no competing interests.

\section{Funding}


The study was funded by the National Center for Women and Children's Health of the Chinese Center for Disease Control and Prevention.

\section{Authors' contributions}

RZ conceived the research. SW, MS, and XH designed the survey. RZ, SW, MS, LY, and DZ implemented the survey. SW and MS conducted the statistical analysis. SW wrote a primary draft and prepared advanced drafts for publication. SW, MS, and XH finalized the report, TX and XP supervised the writing of the report and paper.

\section{Acknowledgments}

We sincerely thank the pregnant women who volunteered to participate in the study. We also thank the hospital staff for their help and the experts who provided valuable suggestions during the study.

\section{References}

1. Cowan PH, M. Family transitions (family research consortium: advances in family research). Hillside, NJ: Lawrence Erlbaum; 1991.

2. WHO. Maternal Mental Health Child Health and Development Literature Review of Risk Factors and Interventions on Postpartum Depression. Geneva.2008.

3. Hahn-Holbrook J, Cornwell-Hinrichs T, Anaya I. Economic and health predictors of National Postpartum Depression Prevalence: a systematic review, meta-analysis, and meta-regression of 291 studies from 56 countries. Frontiers In Psychiatry. 2018;8:248.

4. Sun Mengyun, Huang Xing, Yang yehuan.Natural outcomes and influencing factors anxiety status in Chinese pregnant and parturient women during pregnancy and postpartum. Chinese Journal of Woman and Child Health Research.2021,32(8):1112-1117.

5. Maeve A. O'Connell, Patricia Leahy-Warren, Ali S. Khashan. A systematic review of maternal near miss and mortality due to postpartum hemorrhage. International Journal of Gynecology \& Obstetrics, 2017;137(1):1-7.

6. Staneva AA, Bogossian F, Wittkowski A. The experience of psychological distress, depression, and anxiety during pregnancy: a meta-synthesis of qualitative research. Midwifery. 2015;31(6):563-73.

7. Togher KL, Treacy E, O'Keeffe GW. Maternal distress in late pregnancy alters obstetric outcomes and the expression of genes important for placental glucocorticoid signaling. Psychiatry Res. 2017;255:17-26.

8. Saeed A, Raana T, Saeed AM, Humayun A. Effect of antenatal depression on maternal dietary intake and neonatal outcome: a prospective cohort. Nutr J. 2015;15(1):64.

9. Davis EP, Glynn LM, Waffarn F, Sandman CA. Prenatal maternal stress programs infant stress regulation. J Child Psychol Psychiatry. 2011;52(2):119-29.

10. Alehagen S, Wijma K, Wijma B. Fear during labor. Acta Obstet Gynecol Scand. 2001;80(4):315-20. 
11. Laursen M, Johansen $C$, Hedegaard M. Fear of childbirth and risk for birth complications in nulliparous women in the Danish national birth cohort.Br J Obstet Gynaecol. 2009;116(10):1350-5.

12. Ryding EL, Wijma B, Wijma K, Rydhstrom H. Fear of childbirth during pregnancy may increase the risk of emergency cesarean section. Acta Obstet Gynecol Scand. 1998;77(5):542-7.

13. Lobel M, DeLuca RS. Psychosocial sequelae of cesarean delivery: review and analysis of their causes and implications. Soc Sci Med. 2007;64(11):2272-84.

14. Laursen M, Johansen $C$, Hedegaard M. Fear of childbirth and risk for birth complications in nulliparous women in the Danish national birth cohort. Br J Obstet Gynaecol. 2009;116(10):1350-5.

15. Cooper PJ, Murray L. Postnatal depression. British Medical Journal. 1998;316(7148):1884-6.

16. Dunkel Schetter C. Psychological science on pregnancy: Stress processes, biopsychosocial models, and emerging research issues. Annual Reviews in Psychology.2011; 62, 531-558.

17. Bardacke N. Mindful birthing: training the mind body and heart for childbirth and beyond. New York: Harper One; 2012.

18. Kabat-Zinn J. Mindfulness-based interventions in context: past, present, and future. Clin Psychol Sci Pract. 2003;10(2):144-56.

19. Kabat-Zinn J. Full catastrophe living: using the wisdom of your mind to face stress, pain and illness. New York: Dell Publishing; 1990.

20. Vieten C, Astin J. Effects of a mindfulness-based intervention during pregnancy on prenatal stress and mood: results of a pilot study. Arch Womens Ment Health. 2008;11(1):67-74.

21. Duncan, L. G., \& Bardacke, N. Mindfulness-based childbirth and parenting education: promoting family mindfulness during the perinatal period. Journal of Child \& Family Studies, 2010;19(2), 190202.

22. Bardacke N, Duncan L G.Chapter 10 - mindfulness-based childbirth and parenting: cultivating inner resources for the transition to parenthood and beyond. Mindfulness-Based Treatment Approaches(Second Edition).2014;21.

23. Hofmann SG, Sawyer AT, Witt AA, Oh D. The effect of mindfulness-based therapy on anxiety and depression: a meta-analytic review. J Consult Clin Psychol. 2010;78(2):169-83.

24. Evans S, Ferrando S, Findler M, Stowell C, Smart C, Haglin D. Mindfulness-based cognitive therapy for generalized anxiety disorder. J Anxiety Disord.2008;22(4):716-21.

25. Khoury B, Lecomte T, Fortin G, Masse M, Therien P, Bouchard V, ChapleauM-A, Paquin K, Hofmann SG. Mindfulness-based therapy: a comprehensive meta-analysis. Clin Psychol Rev. 2013;33(6):76371.

26. Nancy Bardacke, Zheng Ruimin.Mindful Birthing.People's Medical Publishing House.2019.

27. Goyal M, Singh S, Sibinga EMS, et al. Meditation programs for psychological stress and well-being: a systematic review and meta-analysis. JAMA Intern Med 2014;174(3):357-68.

28. Cox JL, Chapman G, Murray D, Jones P. Validation of the Edinburgh Postnatal Depression Scale (EPDS) in non-postnatal women. J Affect Disord 1996;39(3):185-9. 
29. Gibson J, McKenzie-McHarg K, Shakespeare J, Price J, Gray R. A systematic review of studies validating the Edinburgh Postnatal Depression Scale in antepartum and postpartum women. Acta Psychiatr Scand 2009;119(5):350-64.

30. Tsao Y, Creedy DK, Gamble J. Prevalence and psychological correlates of postnatal depression in rural Taiwanese women. Health Care Women Int2015;36(4):457-74.

31. Baer RA, Smith GT, Hopkins J, Krietemeyer J, Toney L. Using self-report assessment methods to explore facets of mindfulness. Assessment 2006;13(1):27-45.

32. Diener E, Emmons RA, Larsen RJ, Griffin S. The Satisfaction With Life Scale. J Pers Assess. 1985;49(1):71-5.

33. Wijma K, Wijma B, Zar M. Psychometric aspects of the W-DEQ; a new questionnaire for the measurement of fear of childbirth. J Psychosom Obstet Gynaecol. 1998;19:84-97.

34. Andaroon N, Kordi M, Ghasemi M, Mazlom R. The Validity and Reliability of the Wijma Delivery Expectancy/Experience Questionnaire (Version A) in Primiparous Women in Mashhad, Iran. Iran J Med Sci. 2020;45(2):110-117.

35. Tanaka-Matsumi J, Kameoka VA. Reliabilities and concurrent validities of popular self-report measures of depression, anxiety, and social desirability. J Consult Clin Psychol. 1986;54:328.

36. Zung WWK. A rating instrument for anxiety disorders. Psychosomatics. 1971;12:371-9.

37. Sbrilli MD, Duncan LG, Laurent HK. Effects of prenatal mindfulness-based childbirth education on child-bearers' trajectories of distress: a randomized control trial. BMC Pregnancy Childbirth. 2020;20(1):623.

38. Miklowitz, D., Semple, R., Hauser, M., Elkin, D., Weintraub, M., \&Dimidjian, S.Mindfulness-Based Cognitive Therapy for perinatal women with depression or bipolar spectrum disorder.Cognitive Therapy and Research.2015;39(5),590-600.

39. Luberto, C., Park, E., \& Goodman, J. Postpartum outcomes and formal mindfulness practice in mindfulness-based cognitive therapy for perinatal women.Mindfulness. 2018.;9(3),850-859.

40. Vieten C, Astin J. Effects of a mindfulness-based intervention during pregnancy on prenatal stress and mood: results of a pilot study. Arch Womens Ment Health. 2008;11(1):67-74.

41. Duncan, L. G., Cohn, M. A., Chao, M. T., Cook, J. G., Riccobono, J., \& Bardacke, N. Benefits of preparing for childbirth with mindfulness training: a randomized controlled trial with active comparison. BMC Pregnancy and Childbirth,2017;17(1), 140.

42. Duncan, L. G., \& Bardacke, N. Mindfulness-based childbirth and parenting education: promoting family mindfulness during the perinatal period. Journal of Child \& Family Studies.2010; 19(2), 190202.

43. Veringa IK, de Bruin El, Bardacke N, Duncan LG, van Steensel FJ, Dirksen CD, Bögels SM. 'I've Changed My Mind,' Mindfulness-Based Childbirth and Parenting (MBCP) for pregnant women with a high level of fear of childbirth and their partners: study protocol of the quasi-experimental controlled trial. BMC Psychiatry. 2016;16(1):377. 
44. Byrne, J., Hauck, Y., Fisher, C., Bayes, S., \& Schutze, R. Effectiveness of a mindfulness-based childbirth education pilot study on maternal self-efficacy and fear of childbirth. Journal of Midwifery \& Women's Health.2014; 59(2), 192-197.

45. Veringa-Skiba IK, de Bruin El, van Steensel FJA, Bögels SM. Fear of childbirth, nonurgent obstetric interventions, and newborn outcomes: A randomized controlled trial comparing mindfulness-based childbirth and parenting with enhanced care as usual. Birth. 2021. doi: 10.1111/birt.12571.

46. Byrne J, Hauck Y, Fisher C, Bayes S, Schutze R. Effectiveness of a mindfulness-based childbirth education pilot study on maternal self-efficacy and fear of childbirth. Journal of midwifery \& women's health.2014;59(2):192-7.

47. Kantrowitz-Gordon I, Abbott S, Hoehn R. Experiences of Postpartum Women after Mindfulness Childbirth Classes: A Qualitative Study. J Midwifery Womens Health. 2018;63(4):462-469.

48. Taylor BL, Cavanagh K, Strauss C. The effectiveness of mindfulness-based interventions in the perinatal period: a systematic review and meta-analysis. PLoS One. 2016;11(5):e0155720.

49. Woolhouse H, Mercuri K, Judd F, Brown SJ. Antenatal mindfulness intervention to reduce depression, anxiety and stress: a pilot randomized controlled trial of the Mind Baby Body program in an Australian tertiary maternity hospital. BMC Pregnancy and Childbirth. 2014;14(1):369.

50. Dunn C, Hanieh E, Roberts R, Powrie R. Mindful pregnancy and childbirth: effects of a mindfulnessbased intervention on women's psychological distress and well-being in the perinatal period. Archives of Women's Mental Health. 2012;15(2):139-43.

51. Bowen A, Baetz M, Schwartz L, Balbuena L, Muhajarine N. Antenatal group therapy improves worry and depression symptoms. Isr J Psychiatry Relat Sci.2014;51(3):226-31.

52. Duncan LG, Cohn MA, Chao MT, Cook JG, Riccobono J, Bardacke N. Benefits of preparing for childbirth with mindfulness training: a randomized controlled trial with active comparison. BMC Pregnancy Childbirth.2017;17(1):140.

\section{Figures}




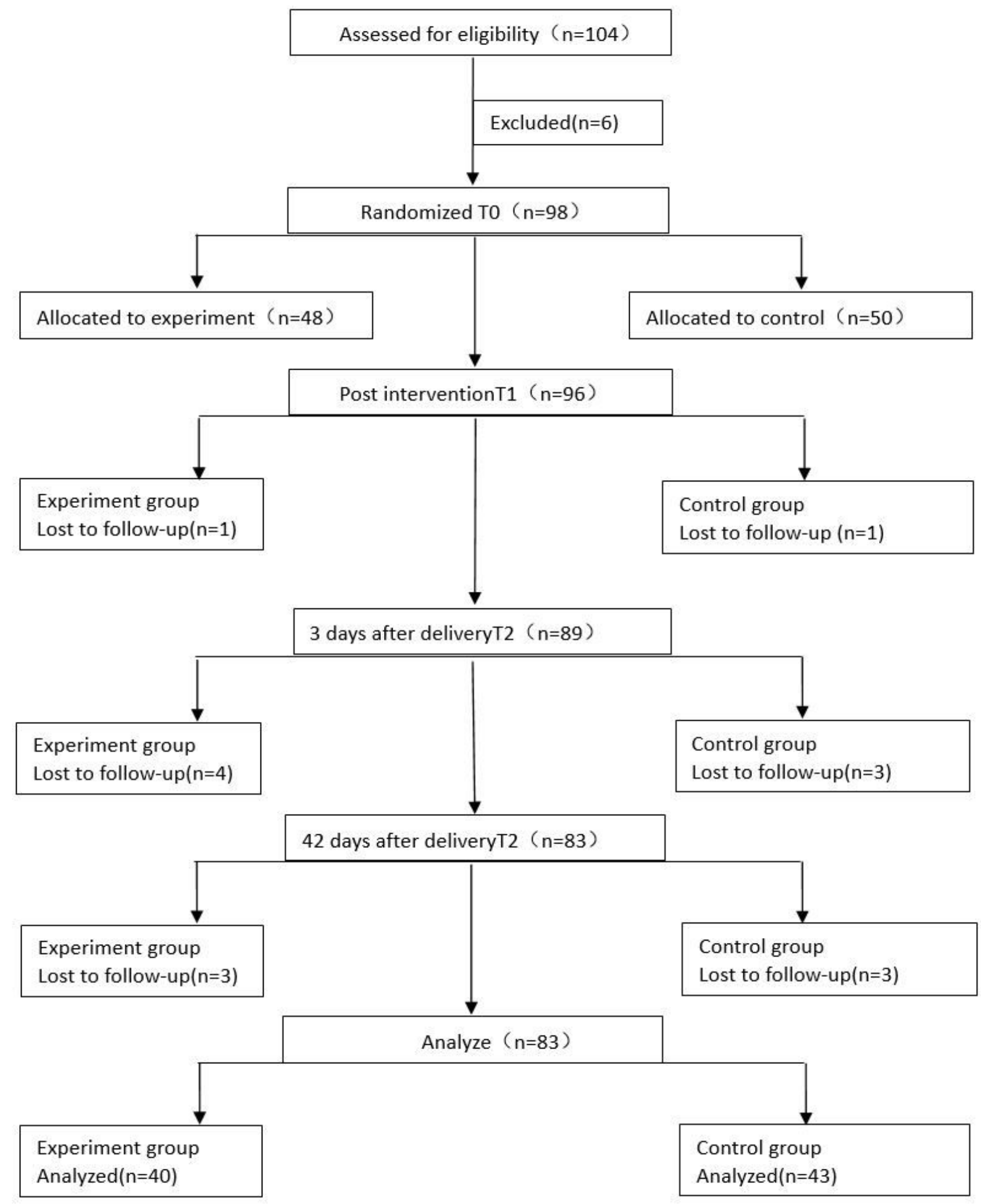

\section{Figure 1}

CONSORT diagram. Passage of participants through each trial stage 

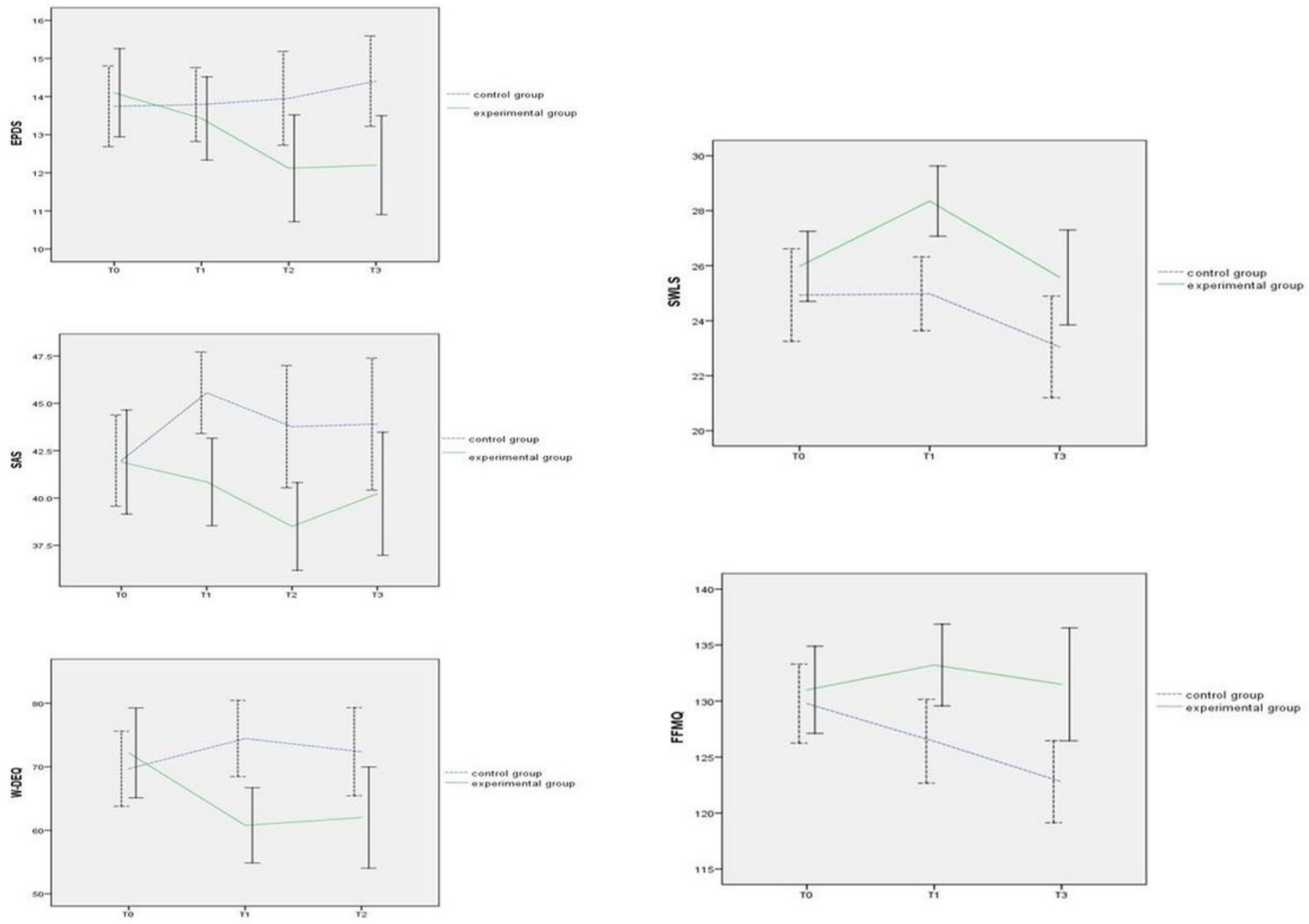

Figure 2

Differences in depression and anxiety at $\mathrm{T} 0, \mathrm{~T} 1, \mathrm{~T} 2$, and $\mathrm{T} 3$; fear and satisfaction with life at $\mathrm{T} 0, \mathrm{~T} 1$, and T3; and mindfulness at T0, T1, and T2. 